\title{
Synthesis of Y-Tip Graphitic Nanoribbons from Alcohol Catalytic Chemical Vapor Deposition on Piezoelectric Substrate
}

\author{
Zainab Yunusa, ${ }^{1,2}$ Suraya Abdul Rashid, ${ }^{1,3}$ Mohd Nizar Hamidon, ${ }^{1}$ \\ Syed Hafiz, ${ }^{4}$ Ismayadi Ismail, ${ }^{1}$ and Saeed Rahmanian ${ }^{5}$ \\ ${ }^{1}$ Institute of Advanced Technology, Universiti Putra Malaysia, 43400 Serdang, Selangor, Malaysia \\ ${ }^{2}$ Bayero University Kano, PMB 3011, Kano, Nigeria \\ ${ }^{3}$ Department of Chemical and Environmental Engineering, Universiti Putra Malaysia, 43400 Serdang, Selangor, Malaysia \\ ${ }^{4}$ Department of Physics, Faculty of Science, Universiti Malaya, 50603 Kuala Lumpur, Malaysia \\ ${ }^{5}$ Department of Mechanical Engineering, Universiti Putra Malaysia, 43400 Serdang, Selangor, Malaysia
}

Correspondence should be addressed to Zainab Yunusa; zee2yunusa@gmail.com

Received 5 March 2015; Accepted 30 April 2015

Academic Editor: Alexander I. Chernov

Copyright (c) 2015 Zainab Yunusa et al. This is an open access article distributed under the Creative Commons Attribution License, which permits unrestricted use, distribution, and reproduction in any medium, provided the original work is properly cited.

\begin{abstract}
We report the synthesis of Graphitic Nanoribbons (GNRs) using Alcohol Catalytic Chemical Vapor Deposition (ACCVD). Bulk GNR was synthesized directly on a piezoelectric substrate using one-step ACCVD. The synthesized GNRs were characterized by X-Ray Diffraction (XRD), Scanning Electron Microscope (SEM), Transmission Electron Microscope (TEM), Energy Dispersive X-Ray (EDX), Atomic Force Microscopy (AFM), and Raman spectroscopy. The characterization results showed Y-tip morphology of bulk and filamentous as-grown GNR having varying width that lies between tens and hundreds of nm and length of several microns. Based on the thickness obtained from the AFM and the analysis from the Raman spectroscopy, it was concluded that the synthesized GNRs are multiple-layered and graphitic in nature. With the direct synthesis of GNR on a piezoelectric substrate, it could have applications in the sensor industries, while the Y-tip GNR could have potentialities in semiconductor applications.
\end{abstract}

\section{Introduction}

Graphitic Nanoribbons (GNRs) are unique materials that belong to the family of carbon nanostructures and were first discovered by Murayama and Maeda in 1990 [1]. They have superior thermal, electronic, and mechanical properties that make them suitable materials in the semiconductor industry and they are predicted to be used together with $\mathrm{Si}$ in the next generation of electronic devices. They also have potential applications in conductive films, sensors, and photonic devices [2].

Different techniques have been employed for the synthesis of highly crystalline GNR. There are the bottom-up approach and the top-down approach. The top-down approach includes lithographic patterning [3], plasma etching of carbon nanotubes (CNT) [4], mechanical exfoliation [5], and sonochemical unzipping of CNT [6-8], while the chemical vapor deposition (CVD) and chemical synthesis are included in the bottom-up approach [9]. Each of these methods produces different GNR with varying widths, thicknesses, and lengths which gives them their unique interesting properties like high mechanical and electronic strengths that are suitable for many applications. Although the top-down approach is widely used for the synthesis of GNR, the disadvantage of these techniques is that it is an indirect method of preparation and therefore the formation and structure of the GNR are solely dependent upon the type of synthesized carbon nanostructure. This makes the control of the GNR structure limited and rather difficult.

Using the CVD technique, the first GNRs were synthesized by Murayama and Maeda [1] from a chemical oxidation/reduction reaction of carbon monoxide at a temperature of $400-700^{\circ} \mathrm{C}$ in the presence of $\mathrm{Fe}(\mathrm{CO})_{5}$ under the flow of $\mathrm{CO} / \mathrm{H}_{2}$ gas. The ribbon-like structures were $10 \mu \mathrm{m}$ in length, $0.1-0.7 \mu \mathrm{m}$ in width, and $10-200 \mathrm{~nm}$ thick, and the catalyst iron nanoparticle was visible at the tip of the ribbon. GNRs 
were also synthesized using CVD technique as reported by Campos-Delgado et al. [10] in which aerosol pyrolysis of ferrocene and thiophene in ethanol was used for the process. Argon was used as a carrier gas and the reaction temperature was $950^{\circ} \mathrm{C}$. Rippled GNRs of micrometers in length, 20$300 \mathrm{~nm}$ in width, and less than $15 \mathrm{~nm}$ thick were produced. Mahanandia et al. [11] also reported the pyrolysis of ferrocene and tetrahydrofuran (THF) at a temperature of $950^{\circ} \mathrm{C}$ to produce GNR and MWNT using iron catalyst. The estimated widths of the ribbons were approximately $200 \mathrm{~nm}$ with length of tens of microns. All these methods require high temperature and do not require any template for the synthesis.

In this paper we report the direct synthesis of long and bulk GNR on piezoelectric substrates which to the best of the authors' knowledge has not been reported in the literature. Gallium orthophosphate $\left(\mathrm{GaPO}_{4}\right)$ which is piezoelectric in nature was chosen due to its stability at high temperature so as to explore its potentialities when employed as a substrate for the synthesis using ACCVD.

\section{Materials and Methods}

2.1. Fabrication of Synthesis Platform. The platform that was used for the synthesis comprises 3 layers: gallium orthophosphate $\left(\mathrm{GaPO}_{4}\right)$ which is a piezoelectric material and zirconium under layer which is sandwiched between the $\mathrm{GaPO}_{4}$ and the platinum. This under layer serves to promote adhesion between the substrate and platinum metal. $\mathrm{GaPO}_{4}$ was chosen as a platform for direct synthesis of the carbon nanostructure. The direct synthesis is one of the techniques used which include spin coating and spraying. Platinum was chosen so as to act as a metal layer for the electrodes when used as a sensor. However, $\mathrm{GaPO}_{4}$ was preferred because future work will focus on the application of GNR for gas sensing in which it would serve as a sensing platform. With the direct synthesis of carbon nanostructure on the $\mathrm{GaPO}_{4}$, there is no need to transfer to another suitable substrate for sensing like $\mathrm{SiO}_{2}$ which makes it relatively simple and economical [12]. Figure 1 shows the cross section of the sample.

2.2. Synthesis of GNR. The substrate was first immersed in acetone so as to remove dust and impurities. After thorough cleansing, $4 \mathrm{~g}$ of iron III nitrate was mixed with $100 \mathrm{~mL}$ of ethanol to form a solution [13]. The solution was stirred gently until the iron nitrate dissolved completely. The substrate was then dip-coated into the prepared solution and left for about 10 minutes. Then, it was left to dry under ambient temperature for 24 hours. After drying, the sample was put in the oven for calcination at a temperature of $200^{\circ} \mathrm{C}$ for 1 hour. The sample was then placed in a ceramic boat and inserted into a horizontal ceramic furnace for the CVD process. The furnace was then heated gradually to a temperature of $700^{\circ} \mathrm{C}$ under argon flow which was set at a flow rate of $100 \mathrm{sccm}$. The reaction took place for a period of 20 minutes under the flow of argon gas and a vapor pressure of $6 \mathrm{mbar}$ for the formation of GNR. Figure 2 shows the illustration of the experimental set-up for the ACCVD. The ethanol serves as the carbon source, while the argon gas serves as the carrier

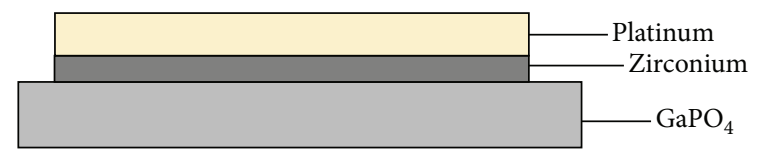

FIGURE 1: Cross section of the sample.

gas. The reaction was stopped and cooled down under argon flow to room temperature. The sample was collected from the ceramic boat and the cross section of the sample is as shown in Figure 2.

2.3. Characterization of GNR. After the sample was collected, different characterizations were carried out for morphological analysis. A Renishaw inVia microscope with different wavelengths of 532 and $633 \mathrm{~nm}$, respectively, and laser power of $1 \mathrm{~mW}$ was used to scan an area of $250 \times 250 \mu \mathrm{m}$ for the Raman spectroscopy analysis. Microscopy analyses for structural and morphological investigations were carried out using Field Emission Scanning Electron Microscope (FESEM, S$3400 \mathrm{~N}$ Hitachi), Transmission Electron Microscope (TEM, LEO 912AB), and High Resolution Transmission Electron Microscope (HRTEM, Libra 200FE). Atomic Force Microscope with noncontact Silicon Cantilever probe NSG10 was used to scan an area of $3 \times 3 \mu \mathrm{m}$ of the GNR to obtain the $2 \mathrm{D}$ topography. The catalyst was also characterized using X-Ray Diffraction (XRD).

\section{Results and Discussions}

3.1. GNR Characterization. The GNR morphology was characterized with FESEM. The micrograph shown in Figure 3(a) revealed the structural morphology of the synthesized bulk Graphitic Nanoribbons. From the micrograph, it can be seen that the GNRs were densely grown, long, and helical and have broad widths ranging from about tens to hundreds of $\mathrm{nm}$. Further characterization was done using TEM to determine the shape and size of the GNR. The image shown in Figure 3 (b) revealed the flat $2 \mathrm{D}$ structure confirming the ribbon-like morphology. The widths of the synthesized GNR correspond well with the FESEM data showing a width range of about tens to hundreds of nm. Figure 3(c) shows a HRTEM image of the sample which revealed a Y-shaped 2D flat structure and also some flat 2D tape-like structures that are transparent and conform with the TEM images of Figure 3(b). The width of the GNR as revealed from the HRTEM is narrow and has a width of few $\mathrm{nm}$.

Energy Dispersive X-Ray Analysis (EDX) was employed for qualitative analysis of the GNR. Based on the EDX analysis, quantitative analysis of the spectrum revealed the percentage constitution of each of the elements present in the sample. carbon constitutes a high percentage of $89.15 \%$ while iron constitutes $10.85 \%$ and the percentage of oxygen is negligible. This shows the high percentage of carbon in the synthesized GNR.

Further characterization was done using AFM in order to obtain the 2D topography and also to determine the thickness 


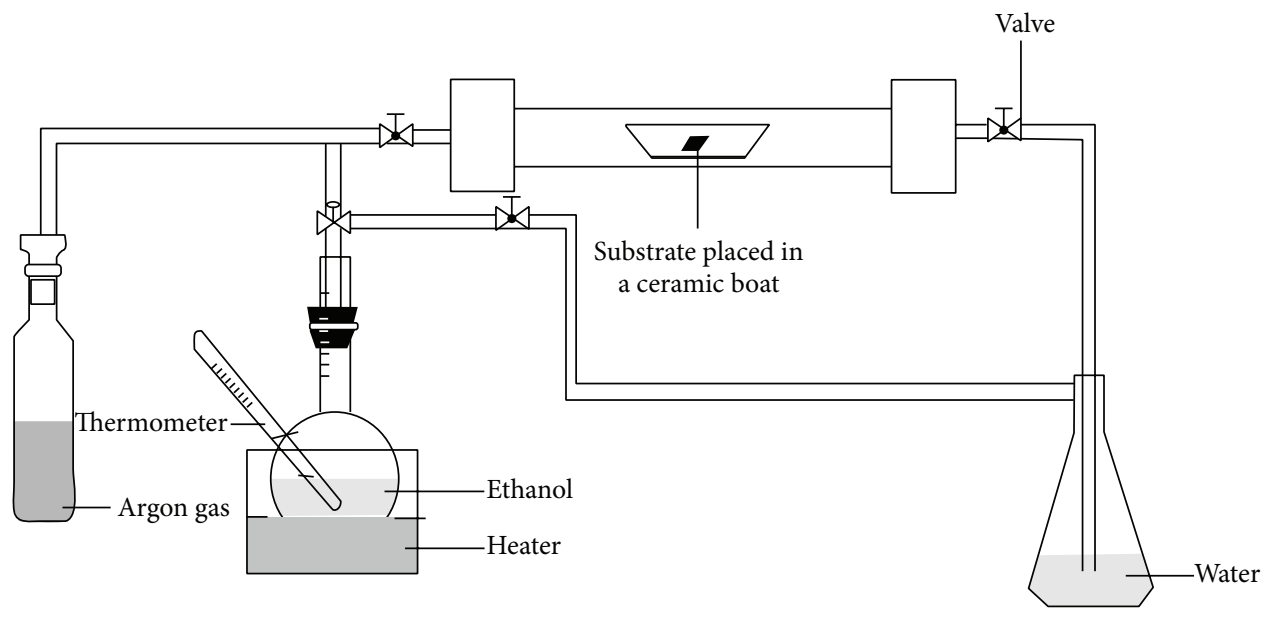

Figure 2: Experimental set-up for ACCVD.

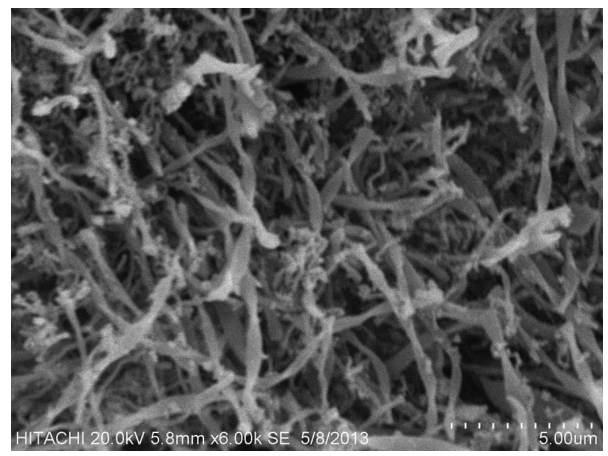

(a)

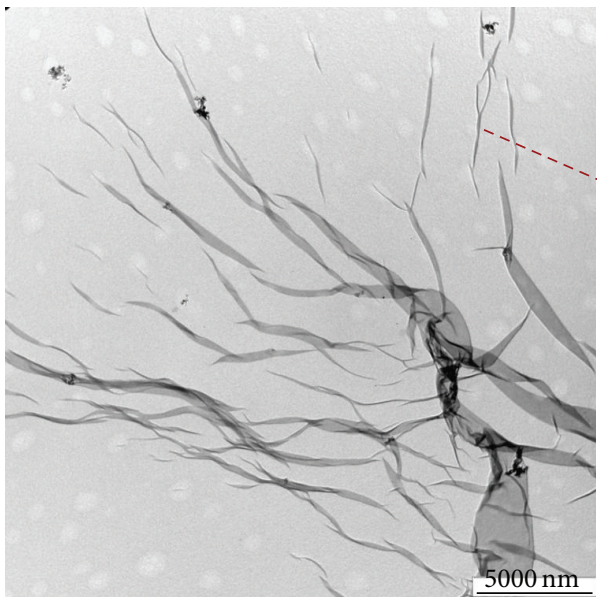

(b)

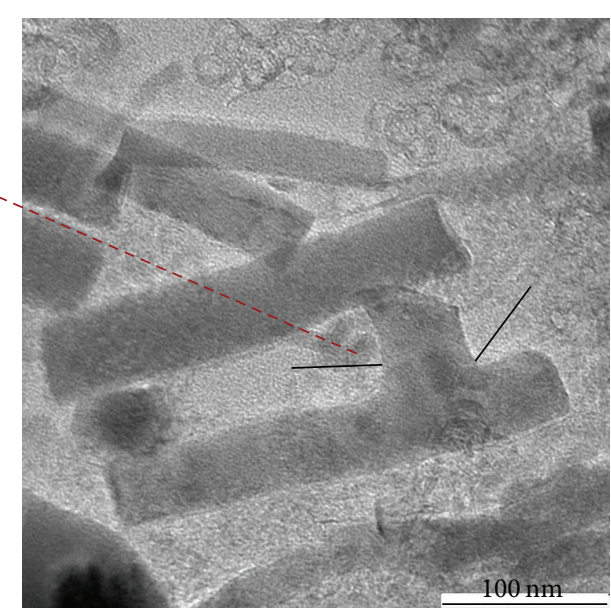

(c)

FIGURE 3: (a) SEM micrograph of the as-grown GNR. (b) TEM image of GNRs showing an inset of Y-tip. (c) HRTEM of GNR showing Y-tip structure as pointed by the black lines.

of the GNR. Figure 4(a) shows an image of an isolated Ytip GNR which is further analyzed as shown in Figure 4(b). Section analysis of the isolated GNR was carried out on two different spots. The section analysis using the AFM revealed that the isolated GNR corresponds to a width of $560 \mathrm{~nm}$.
Since the synthesized GNRs have a width that lies between less than 100 and few hundred $\mathrm{nm}$, this isolated ribbon has a width that corresponds well with the SEM and TEM micrographs shown in Figure 4. Further AFM analysis was carried out on the Y-intersection as shown in Figure 4(b). 

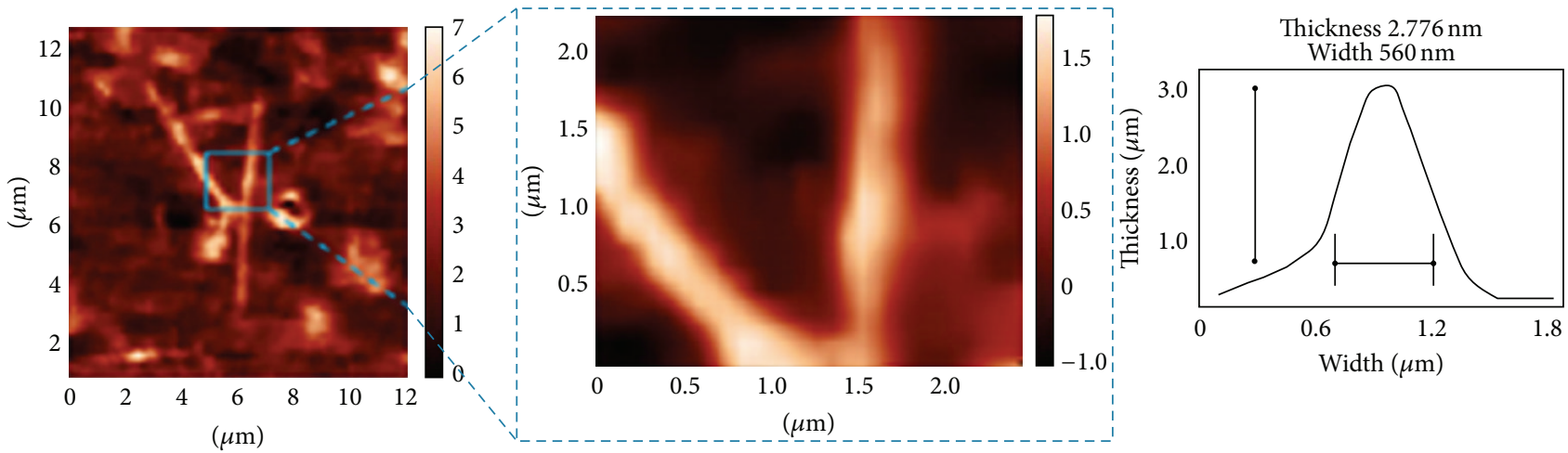

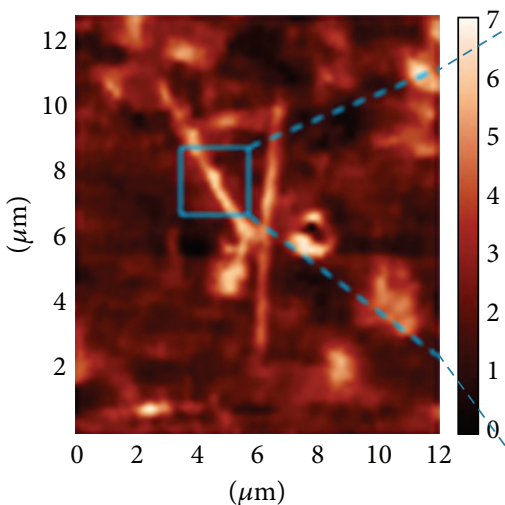

(a)

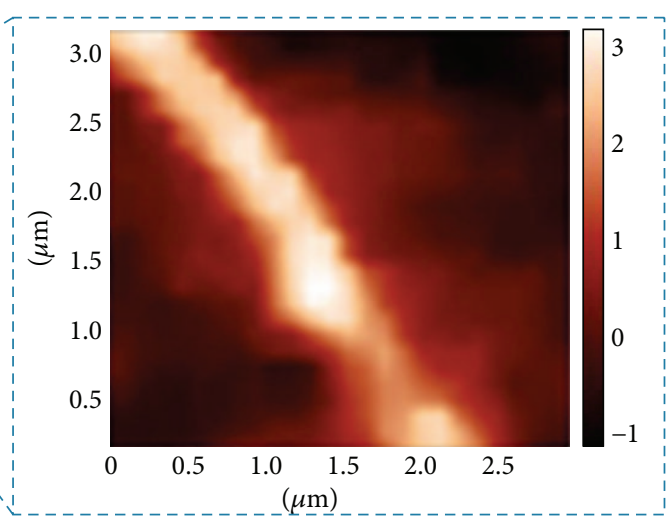

(b)

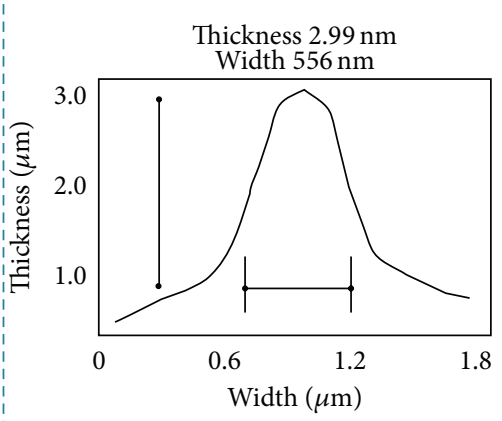

(c)

FIGURE 4: (a) AFM image of an isolated Y-tip GNR, an inset of a GNR spot, and thickness profile of GNR and (b) AFM image of the same GNR on a different spot with the corresponding thickness and (c) thickness profile.

The same ribbon was analyzed but on a different spot which revealed a width of $556 \mathrm{~nm}$ and a corresponding thickness of $2.9 \mathrm{~nm}$.

The topographical curve as shown in Figure 4(a) showed that the GNR with a width of $560 \mathrm{~nm}$ has a corresponding thickness of $2.8 \mathrm{~nm}$. Similarly, the width of the second spot was found to be $556 \mathrm{~nm}$ with a thickness of $3 \mathrm{~nm}$ (Figure 4(b)). As the thickness of a GNR is a measure of number of layers of the ribbon, we can conclude that with a thickness of approximately $3 \mathrm{~nm}$ the GNR have 10 multiple layers assuming a typical $d$-spacing of $0.3 \mathrm{~nm}$ for carbon.

Raman spectroscopy was employed as a tool for qualitative analysis of the as-grown GNR. The Raman spectrum of GNR is depicted in Figure 5. In order to study the effect of the excitation wavelength on the Raman spectra, two different lasers with excitation wavelengths of $532 \mathrm{~nm}$ and $633 \mathrm{~nm}$ were used as shown in the spectra. The same power of $1 \mathrm{~mW}$ was used for both spectra but with different gratings of $2400 \mathrm{I} / \mathrm{mm}$ and $1800 \mathrm{I} / \mathrm{mm}$ for $532 \mathrm{~nm}$ and $633 \mathrm{~nm}$, respectively. With reference to Figure 5, it could be seen that a small peak which is located at about $480 \mathrm{~cm}^{-1}$ is visible and more intense in case of the red laser. The presence of this peak becomes difficult to explain because it has never been reported in Raman spectroscopy of GNR but in analogy to MWNT as shown by $[14,15]$. It could be speculated to arise due to the presence of iron in the synthesis process as shown by [16].

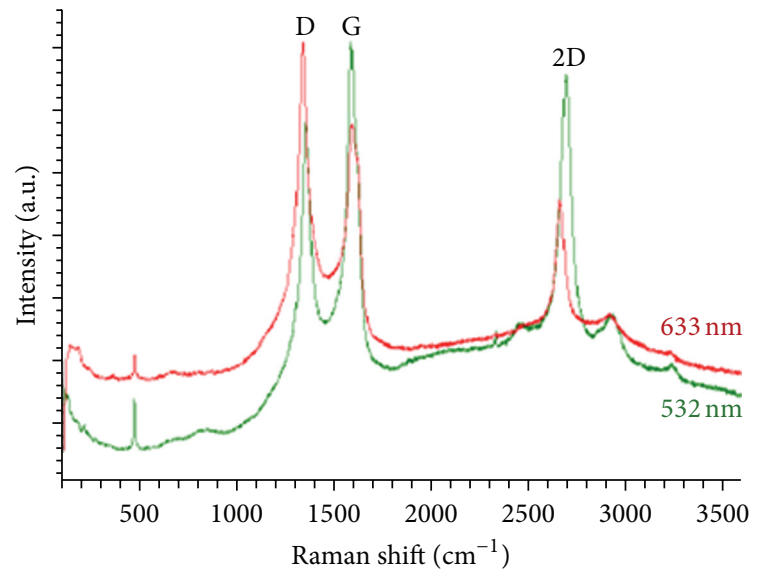

FIGURE 5: Raman spectra of GNR with different laser excitation.

It was also observed that the peak positions of the Dband and G-bands are the same for both laser excitations but their intensities are different. For the case of $633 \mathrm{~nm}$ laser wavelength the D-band intensity is higher than the $532 \mathrm{~nm}$ laser wavelengths. The D-band is a measure of disorder defects in the $\mathrm{sp}^{2}$ carbon. In the case of bulk GNR, there are lots of defects arising from the edges of the ribbon. Similarly, the Gband positions remain the same at $1600 \mathrm{~cm}^{-1}$ but the intensity 


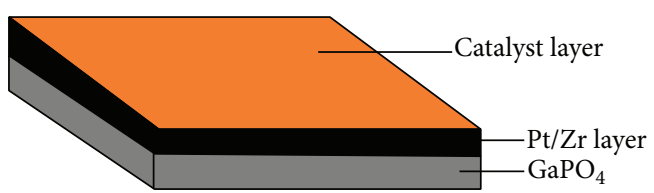

(a)

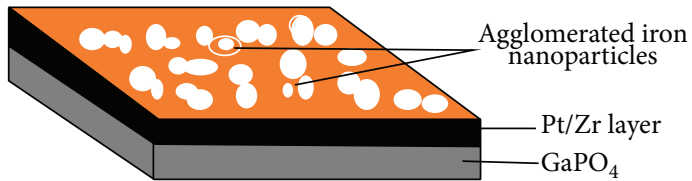

(c)

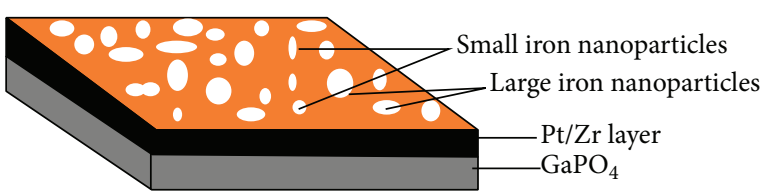

(b)

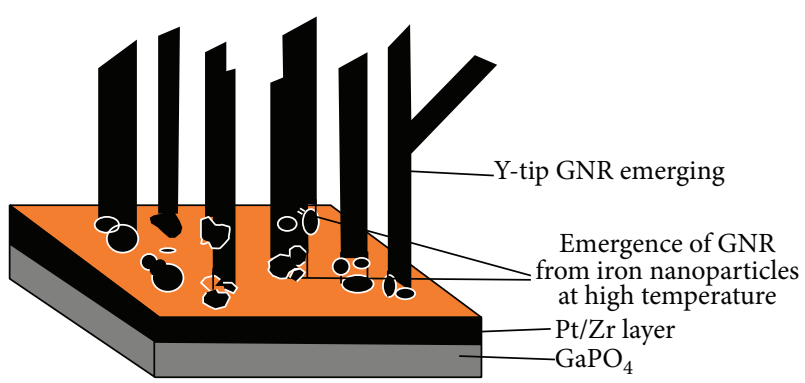

(d)

Figure 6: (a) Substrate after dip-coating in catalyst solution. (b) After calcination at $200^{\circ} \mathrm{C}$ iron nanoparticles are formed. (c) The iron particles agglomerate to form bundles of different diameters. (d) The agglomerated iron nanoparticles emerge into GNR in the presence of argon as carrier gas and ethanol as the carbon feedstock at high temperature. The GNRs emerge into different widths due to nonuniform diameters of the iron nanoparticles.

of the $532 \mathrm{~nm}$ wavelength is greater than that of $633 \mathrm{~nm}$. The G-band is a measure of the graphitic nature and crystallinity of the $\mathrm{sp}^{2}$ hybridized carbon. Another band called the $2 \mathrm{D}$ band is well pronounced and is described as a fingerprint for GNR [17]. The position of the 2D band is different for the two lasers, with $532 \mathrm{~nm}$ at $2700 \mathrm{~cm}^{-1}$ position and $633 \mathrm{~nm}$ lasers at $2650 \mathrm{~cm}$ position.

Raman spectroscopy analysis was carried out on an area of $250 \times 250$ microns. However, since the ribbons are of varying widths and the analysis was done in a specified area, it shows that it was not done on a single isolated nanoribbon. It is speculated that these varying widths and thicknesses could also lead to different crystallinity behaviors as shown in the significant difference of the $\mathrm{I}_{\mathrm{D}} / \mathrm{I}_{\mathrm{G}}$ ratios with different laser excitation. The $\mathrm{I}_{\mathrm{D}} / \mathrm{I}_{\mathrm{G}}$ ratio is used to determine the crystallinity of carbon nanostructures: the higher it is, the more defects are present in the structures. Therefore, from Figure 5 it could be seen that the $\mathrm{I}_{\mathrm{D}} / \mathrm{I}_{\mathrm{G}}$ ratios are 0.7 and 1.03 for $532 \mathrm{~nm}$ and $633 \mathrm{~nm}$ wavelengths excitation, respectively. This shows that the $\mathrm{I}_{\mathrm{D}} / \mathrm{I}_{\mathrm{G}}$ ratio for $532 \mathrm{~nm}$ showed a higher degree of crystallinity.

Similarly, the $\mathrm{I}_{2 \mathrm{D}} / \mathrm{I}_{\mathrm{G}}$ ratios were computed to be 1.03 and 1.04 for $532 \mathrm{~nm}$ and $633 \mathrm{~nm}$ laser wavelengths, respectively. As reported by [17] since the $\mathrm{I}_{2 \mathrm{D}} / \mathrm{I}_{\mathrm{G}}$ ratio is used to investigate the number of layers, then from our data we can conclude that with a ratio of 1.03 our ribbons are multiple-layered in nature and are less defective with an $\mathrm{I}_{\mathrm{D}} / \mathrm{I}_{\mathrm{G}}$ ratio of 0.7 . The multiplelayered characteristic of our GNR could be supported by the work from other authors, whereby few layered GNRs were synthesized using $\mathrm{CVD}$ but with $\mathrm{Cu}$ as the catalyst precursor. In their work, few-layered Graphitic Nanoribbons were synthesized directly on a dielectric surface using CVD and their $\mathrm{I}_{2 \mathrm{D}} / \mathrm{I}_{\mathrm{G}}$ ratio was found to be 0.7 [12]. It could be seen that we have a small peak corresponding to the $D+G$ band at $2940 \mathrm{~cm}^{-1}$ and the intensity is higher for the green laser and almost negligible for the red laser. Another small peak at $3240 \mathrm{~cm}^{-1}$ which is described as $2 \mathrm{D}^{\prime}$ peak is visible and more pronounced in the green laser than in the red laser. The high value in this case could be a result of the bulk synthesis of the ribbons with varying widths because the area selected consisted of bulk GNR with varying widths and not on an isolated ribbon. Due to the defects resulting from the edges of the bulk GNR, the presence of the high intensity D-band is clearly visible.

According to the obtained results, a hypothesis of the growth mechanism of GNR can be explained as follows (Figure 6). The mechanism of the growth is that during calcination the iron nitrate starts to decompose to form iron particles. However, the size of these iron nanoparticles is not homogeneous. With continuous heating, the nanoparticles agglomerate and transform into different iron crystal phase and orientation. Some crystals become cubical whilst others become pyramidal as indicated by the SEM micrograph shown in Figures 7(a) and 7(b). During synthesis these iron nanoparticles agglomerate and form bundles of different clusters of diameters which emerged into GNRs of varying widths and thicknesses.

The prepared samples were characterized with an XRD so as to obtain the spectrum and reveal the crystal orientation and phase of the catalyst precursor deposited on the substrate by dip-coating technique on the substrate. The prepared catalyst was analyzed by XRD analysis in order to identify the active oxide phases which may contribute to catalytic activity. The type of metal oxide in the catalyst is one of the factors that influence the catalytic activity and morphology of the deposited carbon [18] in the synthesis of GNR. The XRD spectrum in Figure 8 showed that the degree of crystallinity of the catalyst was very high. XRD diffractogram revealed the presence of Fe with a cubic crystal phase in the catalyst at $2 \theta$ around $44.6^{\circ}$ and $65.02^{\circ}$. 


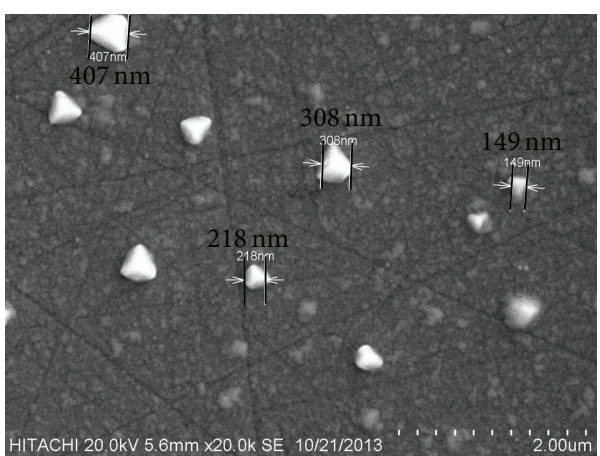

(a)

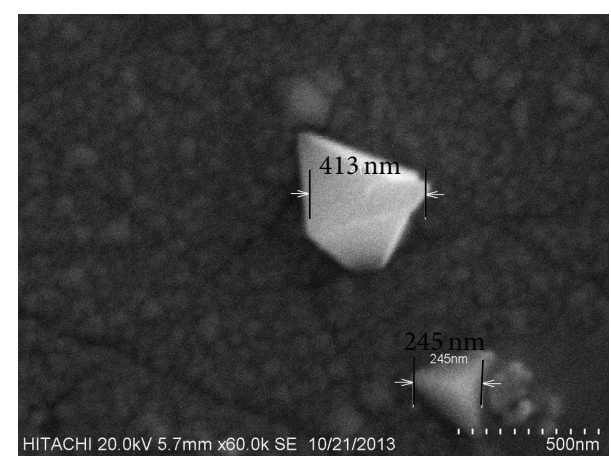

(b)

FIGURE 7: (a) SEM micrograph of iron crystal catalyst after heating (low magnification). (b) SEM micrograph of iron crystal catalyst (high magnification).

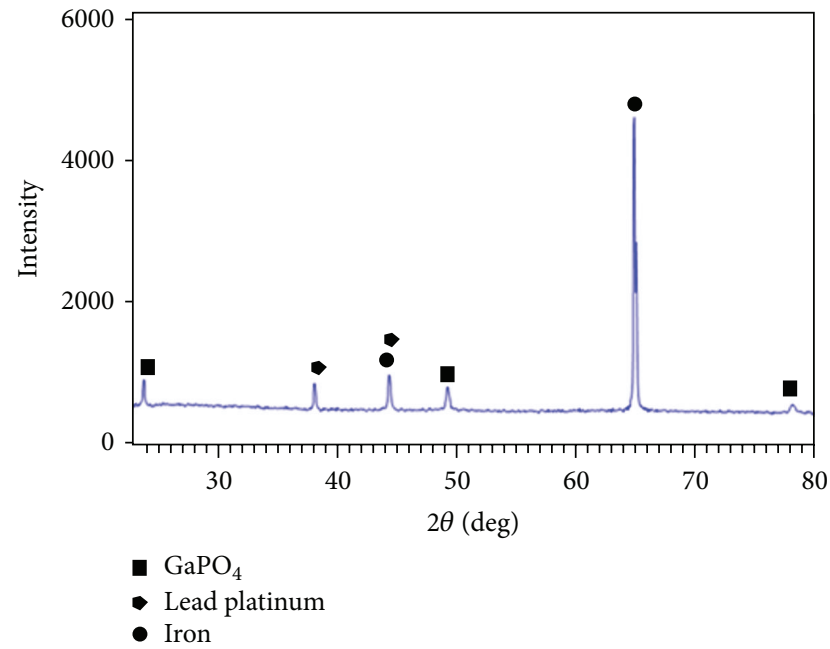

FIGURE 8: XRD spectrum of iron catalyst after dip-coating of the substrate and calcination at $700^{\circ} \mathrm{C}$.

Besides, Fe has been recognized as the domain species in the growth of GNR based on the high intensity peak of the XRD diffractogram, while a low detection on another peak is detected which is attributed to the hexagonal $\mathrm{GaPO}_{4}$ at $2 \theta$ around $24.14^{\circ}$ and $49.44^{\circ}$. The average crystallite size of Fe was calculated using the Debye-Scherrer formula [17]. The average crystallite size of iron nanoparticles was determined as about $27.8 \mathrm{~nm}$. On the other hand, platinum has been identified as lead platinum in the sample. Since the intensity of the peak is low, thus the contribution of platinum as catalyst in the direct formation of GNR is very low. This can be related to the low carbon solubility possessed by platinum which has effect on the width or diameter of the carbon nanoparticles. It is speculated that our Y-tip GNR emerged from cubic crystal iron nanoparticles based on the analysis of the XRD findings. Since the morphology and structure of the final product are determined by the catalyst, the formation of the Y-tip GNR could be supported by [19] in which Y-branched carbon fibres were synthesized with nickel nitrate and iron nitrate as the catalyst precursors.
The crystal phase of the iron crystal is cubical as revealed from the XRD analysis (Figure 8). However, $\mathrm{GaPO}_{4}$ is also a favorable substrate for the formation of the cubical iron crystal structure due to hexagonal crystal structure as revealed from the XRD findings. Subsequently when the ethanol is purged during the reaction, these iron nanoparticles grow from the base and tip to form long tape-like and Y-branched structure and the GNRs emerge with different widths due to the nonhomogeneity of the diameters of the iron nanoparticles.

\section{Conclusion}

The synthesis of bulk GNR directly on a piezoelectric substrate using the ACCVD was reported. Characterizations of the as-grown ribbons on gallium orthophosphate revealed a homogeneous growth of bulk GNRs with varying widths ranging from a few $\mathrm{nm}$ to hundreds of $\mathrm{nm}$ and few microns in length. Raman spectroscopy was employed as a tool for qualitative analysis and the result showed a value of $\mathrm{I}_{\mathrm{D}} / \mathrm{I}_{\mathrm{G}}$ ratio of 0.75 indicating a highly crystalline GNR. Thus, the effect of different laser excitation was investigated. It could be deduced that the lower the laser excitation wavelength, the lesser the $\mathrm{I}_{\mathrm{D}} / \mathrm{I}_{\mathrm{G}}$ ratio and the higher the $\mathrm{I}_{2 \mathrm{D}} / \mathrm{I}_{\mathrm{G}}$ ratio obtained. Therefore, we can conclude that our ribbons are multiple-layered due to the high intensity of the $2 \mathrm{D}$ peak with an $\mathrm{I}_{2 \mathrm{D}} / \mathrm{I}_{\mathrm{G}}$ of 1.03 which is in good agreement with the AFM profiling indicating a ribbon width of $2.776 \mathrm{~nm}$ and $2.990 \mathrm{~nm}$, respectively. For quantitative analysis, Energy Dispersive XRay Analysis was carried out and the result showed high percentage composition of carbon. XRD characterization of catalyst showed that the crystal symmetry of iron nanoparticles is cubical and this could be used to explain why the Y-tip GNR emerged from the synthesis. With the successful synthesis on a piezoelectric substrate, future work will focus on exploring the potential application of the GNR as an active material in the sensor industry and semiconductor application.

\section{Conflict of Interests}

The authors declare that there is no conflict of interests regarding the publication of this paper. 


\section{Acknowledgments}

The authors wish to thank the Ministry of Science, Technology and Innovation (MOSTI), Malaysia, for the grant provided (5450602) and UPM grant (9421300).

\section{References}

[1] H. M. Murayama and T. Maeda, "A novel form of filamentous graphite," Nature, vol. 345, no. 6278, pp. 791-793, 1990.

[2] L. Ma, J. Wang, and F. Ding, "Recent progress and challenges in graphene nanoribbon synthesis," ChemPhysChem, vol. 14, no. 1, pp. 47-54, 2013.

[3] J. Cai, P. Ruffieux, R. Jaafar et al., "Atomically precise bottomup fabrication of graphene nanoribbons," Nature, vol. 466, no. 7305, pp. 470-473, 2010.

[4] L. Jiao, L. Zhang, X. Wang, G. Diankov, and H. Dai, "Narrow graphene nanoribbons from carbon nanotubes," Nature, vol. 458, no. 7240, pp. 877-880, 2009.

[5] L. Jiao, X. Wang, G. Diankov, H. Wang, and H. Dai, "Facile synthesis of high-quality graphene nanoribbons," Nature Nanotechnology, vol. 5, no. 5, pp. 321-325, 2010.

[6] D. V. Kosynkin, A. L. Higginbotham, A. Sinitskii et al., "Longitudinal unzipping of carbon nanotubes to form graphene nanoribbons," Nature, vol. 458, no. 7240, pp. 872-876, 2009.

[7] W. Yoon, Y. Lee, H. Jang et al., "Graphene nanoribbons formed by a sonochemical graphene unzipping using flavin mononucleotide as a template," Carbon, vol. 81, pp. 629-638, 2015.

[8] N. Zhuang, C. Liu, L. Jia et al., "Clean unzipping by steam etching to synthesize graphene nanoribbons," Nanotechnology, vol. 24, no. 32, Article ID 325604, 2013.

[9] Y. Fan, J. Li, X. Liu et al., "A microexplosion method for the synthesis of graphene nanoribbons," Carbon, vol. 49, no. 4, pp. 1439-1445, 2011.

[10] J. Campos-Delgado, J. M. Romo-Herrera, X. Jia et al., "Bulk production of a new form of sp 2 carbon: crystalline graphene nanoribbons," Nano Letters, vol. 8, no. 9, pp. 2773-2778, 2008.

[11] P. Mahanandia, K. K. Nanda, V. Prasad, and S. V. Subramanyam, "Synthesis and characterization of carbon nanoribbons and single crystal iron filled carbon nanotubes," Materials Research Bulletin, vol. 43, no. 12, pp. 3252-3262, 2008.

[12] T. Kaplas, D. Sharma, and Y. Svirko, "Few-layer graphene synthesis on a dielectric substrate," Carbon, vol. 50, no. 4, pp. 1503-1509, 2012.

[13] S. Rahmanian, A. R. Suraya, R. Zahari, and E. S. Zainudin, "Synthesis of vertically aligned carbon nanotubes on carbon fiber," Applied Surface Science, vol. 271, pp. 424-428, 2013.

[14] R. A. DiLeo, B. J. Landi, and R. P. Raffaelle, "Purity assessment of multiwalled carbon nanotubes by Raman spectroscopy," Journal of Applied Physics, vol. 101, no. 6, Article ID 064307, 2007.

[15] L. Bokobza and J. Zhang, "Raman spectroscopic characterization of multiwall carbon nanotubes and of composites," Express Polymer Letters, vol. 6, no. 7, pp. 601-608, 2012.

[16] J. H. Lehman, M. Terrones, E. Mansfield, K. E. Hurst, and V. Meunier, "Evaluating the characteristics of multiwall carbon nanotubes," Carbon, vol. 49, no. 8, pp. 2581-2602, 2011.

[17] L. M. Malard, M. A. Pimenta, G. Dresselhaus, and M. S. Dresselhaus, "Raman spectroscopy in graphene," Physics Reports, vol. 473, no. 5-6, pp. 51-87, 2009.

[18] V. O. Nyamori, S. D. Mhlanga, and N. J. Coville, "The use of organometallic transition metal complexes in the synthesis of shaped carbon nanomaterials," Journal of Organometallic Chemistry, vol. 693, no. 13, pp. 2205-2222, 2008.

[19] L. I. Fe and Z. O. U. Wj, "Synthesis of Y-junction carbon nanofibres by ethanol catalytic combustion technique B," Transactions of Nonferrous Metals Society of China, vol. 16, pp. 431-434, 2007. 

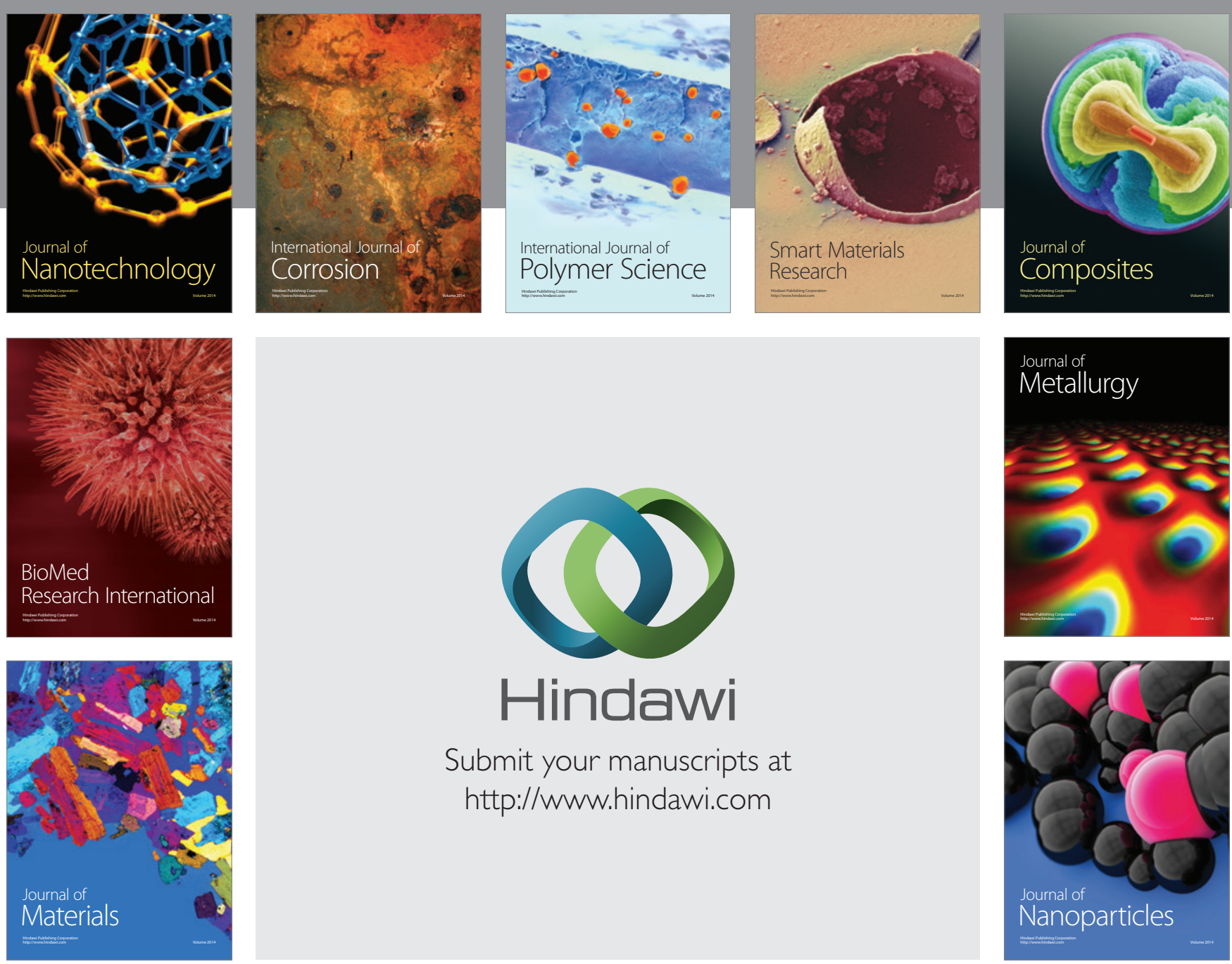

Submit your manuscripts at http://www.hindawi.com
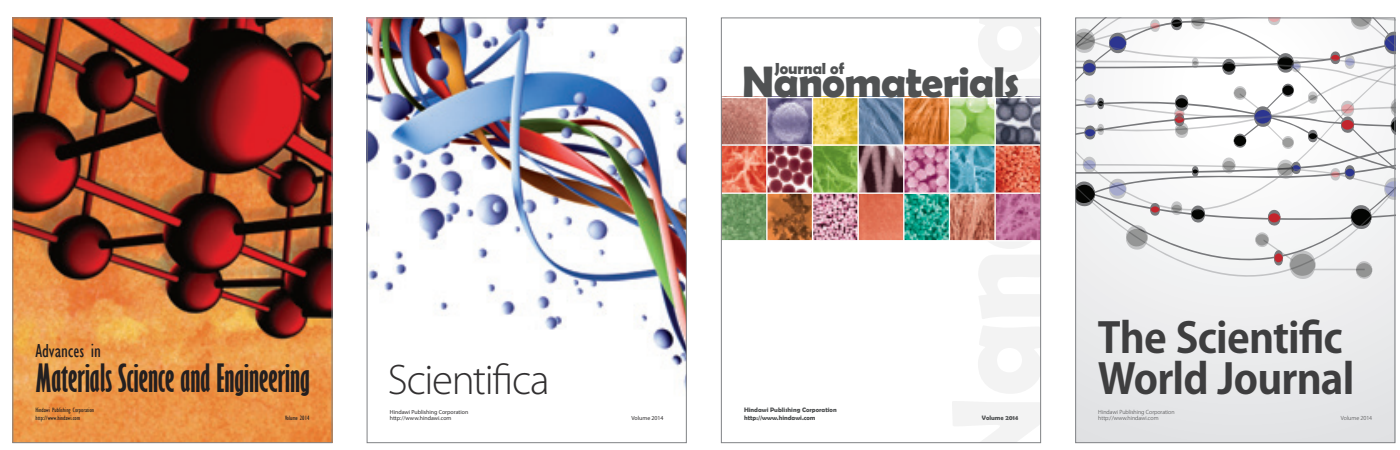

\section{The Scientific World Journal}
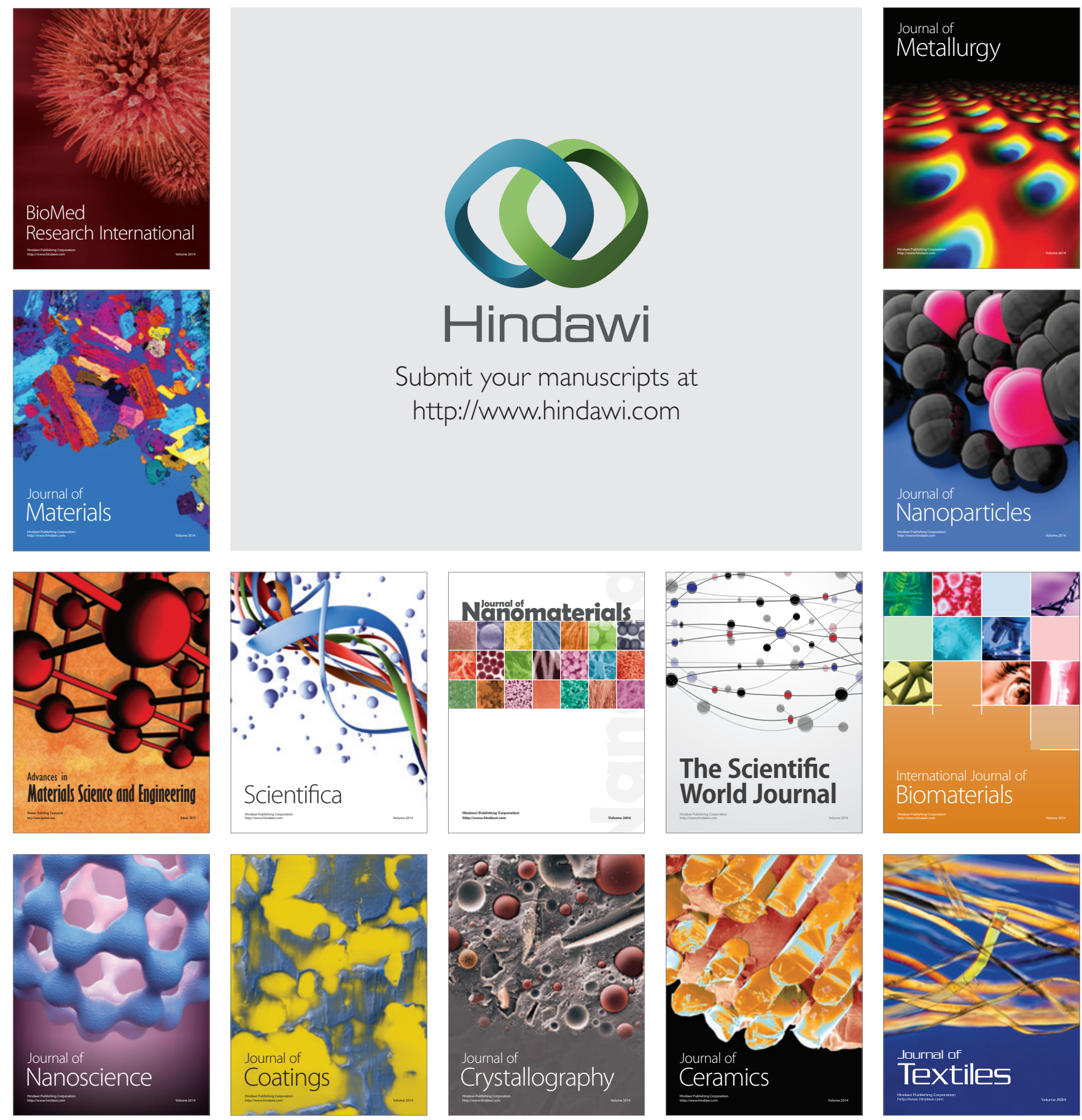